\title{
Derivation of Commutative Rings and the Leibniz Formula for Power of Derivation
}

\author{
Yasushige Watase \\ Suginami-ku Matsunoki \\ 3-21-6 Tokyo, Japan
}

Summary. In this article we formalize in Mizar 1, 2] a derivation of commutative rings, its definition and some properties. The details are to be referred to [5], 7]. A derivation of a ring, say $D$, is defined generally as a map from a commutative ring $A$ to $A$-Module $M$ with specific conditions. However we start with simpler case, namely dom $D=\operatorname{rng} D$. This allows to define a derivation in other rings such as a polynomial ring.

A derivation is a map $D: A \longrightarrow A$ satisfying the following conditions:

(i) $D(x+y)=D x+D y$,

(ii) $D(x y)=x D y+y D x, \forall x, y \in A$.

Typical properties are formalized such as:

$$
D\left(\sum_{i=1}^{n} x_{i}\right)=\sum_{i=1}^{n} D x_{i}
$$

and

$$
D\left(\prod_{i=1}^{n} x_{i}\right)=\sum_{i=1}^{n} x_{1} x_{2} \cdots D x_{i} \cdots x_{n}\left(\forall x_{i} \in A\right) .
$$

We also formalized the Leibniz Formula for power of derivation $D$ :

$$
D^{n}(x y)=\sum_{i=0}^{n}\left(\begin{array}{l}
n \\
i
\end{array}\right) D^{i} x D^{n-i} y .
$$

Lastly applying the definition to the polynomial ring of $A$ and a derivation of polynomial ring was formalized. We mentioned a justification about compatibility of the derivation in this article to the same object that has treated as differentiations of polynomial functions 3 .

MSC: 13B25 13N15 68V20

Keywords: derivation; Leibniz Formula; derivation of polynomial ring MML identifier: RINGDER1, version: 8.1.11 5.65.1394 


\section{Preliminaries}

From now on $L$ denotes an Abelian, left zeroed, add-associative, associative, right zeroed, right complementable, distributive, non empty double loop structure, $a, b, c$ denote elements of $L, R$ denotes a non degenerated commutative ring, and $n, m, i, j, k$ denote natural numbers.

Now we state the propositions:

(1) $n \cdot a+n \cdot b=n \cdot(a+b)$.

Proof: Define $\mathcal{P}$ [natural number] $\equiv \$_{1} \cdot a+\$_{1} \cdot b=\$_{1} \cdot(a+b)$. For every natural number $n$ such that $\mathcal{P}[n]$ holds $\mathcal{P}[n+1]$. For every natural number $n, \mathcal{P}[n]$.

(2) $(n \cdot a) \cdot b=a \cdot(n \cdot b)$.

Proof: Define $\mathcal{P}$ [natural number $] \equiv\left(\$_{1} \cdot a\right) \cdot b=a \cdot\left(\$_{1} \cdot b\right)$. For every natural number $n$ such that $\mathcal{P}[n]$ holds $\mathcal{P}[n+1]$. For every natural number $n, \mathcal{P}[n]$.

(3) $n \cdot\left(0_{L}\right)=0_{L}$.

Proof: Define $\mathcal{P}$ [natural number] $\equiv \$_{1} \cdot\left(0_{L}\right)=0_{L}$. For every natural number $n$ such that $\mathcal{P}[n]$ holds $\mathcal{P}[n+1]$. For every natural number $n$, $\mathcal{P}[n]$.

(4) $0_{L} \cdot n=0_{L}$.

Proof: Define $\mathcal{P}$ [natural number $] \equiv 0_{L} \cdot \$_{1}=0_{L}$. For every natural number $n$ such that $\mathcal{P}[n]$ holds $\mathcal{P}[n+1]$. For every natural number $n, \mathcal{P}[n]$.

\section{Definition of Derivation of Rings and its Properties}

From now on $D$ denotes a function from $R$ into $R$ and $x, y, z$ denote elements of $R$.

Definition of derivation of rings.

Let us consider $R$. Let $\Delta$ be a function from $R$ into $R$. We say that $\Delta$ is derivation if and only if

(Def. 1) for every elements $x, y$ of $R, \Delta(x+y)=\Delta(x)+\Delta(y)$ and $\Delta(x \cdot y)=$ $x \cdot \Delta(y)+y \cdot \Delta(x)$.

Observe that every function from $R$ into $R$ which is derivation is also additive and there exists a function from $R$ into $R$ which is derivation.

A derivation of $R$ is derivation function from $R$ into $R$. The functor Der $R$ yielding a subset of $\left(\Omega_{R}\right)^{\Omega_{R}}$ is defined by the term

(Def. 2) $\{f$, where $f$ is a function from $R$ into $R: f$ is derivation $\}$. 
Let us observe that Der $R$ is non empty.

From now on $D$ denotes a derivation of $R$.

Now we state the propositions:

(5)

(i) $D\left(1_{R}\right)=0_{R}$, and

(ii) $D\left(0_{R}\right)=0_{R}$.

(6) $D(n \cdot x)=n \cdot D(x)$.

Proof: Define $\mathcal{P}$ [natural number $] \equiv D\left(\$_{1} \cdot x\right)=\$_{1} \cdot D(x)$. For every natural number $n$ such that $\mathcal{P}[n]$ holds $\mathcal{P}[n+1]$. For every natural number $n, \mathcal{P}[n]$.

(7) $D\left(x^{m+1}\right)=(m+1) \cdot\left(x^{m} \cdot D(x)\right)$.

Proof: Define $\mathcal{P}$ [natural number $] \equiv D\left(x^{\$_{1}+1}\right)=\left(\$_{1}+1\right) \cdot\left(x^{\$_{1}} \cdot D(x)\right)$. For every natural number $n$ such that $\mathcal{P}[n]$ holds $\mathcal{P}[n+1]$. For every natural number $n, \mathcal{P}[n]$.

(8) (i) $D^{n+1}=D \cdot\left(D^{n}\right)$, and

(ii) $\operatorname{dom} D=$ the carrier of $R$, and

(iii) $\operatorname{dom}\left(D^{n}\right)=$ the carrier of $R$, and

(iv) $D^{n}$ is a (the carrier of $R$ )-valued function.

(9) $\left(D^{n+1}\right)(x)=D\left(\left(D^{n}\right)(x)\right)$. The theorem is a consequence of $(8)$.

(10) If $z \cdot y=1_{R}$, then $y^{2} \cdot D(x \cdot z)=y \cdot D(x)-x \cdot D(y)$.

In the sequel $s$ denotes a finite sequence of elements of the carrier of $R$ and $h$ denotes a function from $R$ into $R$.

Let us consider $R, s$, and $h$. One can check that the functor $h \cdot s$ yields a finite sequence of elements of the carrier of $R$. Now we state the proposition:

(11) If $h$ is additive, then $h\left(\sum s\right)=\sum(h \cdot s)$.

Proof: Define $\mathcal{P}$ [natural number] $\equiv$ for every $h$ and $s$ such that len $s=\$_{1}$ and $h$ is additive holds $h\left(\sum s\right)=\sum(h \cdot s) . \mathcal{P}[0]$ by [4, (6)]. For every natural number $n$ such that $\mathcal{P}[n]$ holds $\mathcal{P}[n+1]$. For every natural number $n, \mathcal{P}[n]$.

(12) Formula $\left(f_{1}+f_{2}+\cdots+f_{n}\right)^{\prime}=f_{1}^{\prime}+f_{2}^{\prime}+\cdots+f_{n}^{\prime}$ : $D\left(\sum s\right)=\sum(D \cdot s)$.

Let us consider $R, D$, and $s$. The functor $\operatorname{DProd}(D, s)$ yielding a finite sequence of elements of the carrier of $R$ is defined by

(Def. 3) len $i t=\operatorname{len} s$ and for every $i$ such that $i \in \operatorname{dom} i t$ holds $i t(i)=$ $\prod \operatorname{Replace}\left(s, i, D\left(s_{/ i}\right)\right)$.

Now we state the propositions:

(13) If len $s=1$, then $\sum \operatorname{DProd}(D, s)=D\left(\prod s\right)$. 
(14) Let us consider a finite sequence $t$ of elements of the carrier of $R$. If len $t \geqslant 1$, then $\sum \operatorname{DProd}(D, t)=D\left(\prod t\right)$.

Proof: Define $\mathcal{P}$ [non zero natural number] $\equiv$ for every $s$ such that len $s=$ $\$_{1}$ holds $\sum \operatorname{DProd}(D, s)=D\left(\prod s\right)$. $\mathcal{P}[1]$. For every non zero natural number $k$ such that $\mathcal{P}[k]$ holds $\mathcal{P}[k+1]$. For every non zero natural number $k, \mathcal{P}[k]$.

\section{Proof of the Leibniz Formula for Power of Derivations}

The Leibniz formula for power of a derivation of a commutative ring.

Let us consider $R, D$, and $n$. Let $x, y$ be elements of $R$. The functor $\mathrm{LBZ}(D, n, x, y)$ yielding a finite sequence of elements of the carrier of $R$ is defined by

(Def. 4) len $i t=n+1$ and for every $i$ such that $i \in \operatorname{dom} i t$ holds $i t(i)=\left(\begin{array}{c}n \\ i-^{\prime} 1\end{array}\right)$. $\left(D^{n+1-^{\prime} i}\right)(x) \cdot\left(D^{i-^{\prime}} 1\right)(y)$.

Now we state the propositions:

(15) $\operatorname{LBZ}(D, 0, x, y)=\langle x \cdot y\rangle$.

(16) $\operatorname{LBZ}(D, 1, x, y)=\langle y \cdot D(x), x \cdot D(y)\rangle$.

Let us consider $R, D$, and $m$. Let $x, y$ be elements of $R$. The functor $\operatorname{LBZ0}(D, m, x, y)$ yielding a finite sequence of elements of the carrier of $R$ is defined by

(Def. 5) len $i t=m$ and for every $i$ such that $i \in \operatorname{dom} i t$ holds $i t(i)=\left(\left(\begin{array}{c}m \\ i-^{\prime} 1\end{array}\right)+\right.$ $\left.\left(\begin{array}{c}m \\ i\end{array}\right)\right) \cdot\left(D^{m+1-^{\prime} i}\right)(x) \cdot\left(D^{i}\right)(y)$.

The functor $\operatorname{LBZ1}(D, m, x, y)$ yielding a finite sequence of elements of the carrier of $R$ is defined by

(Def. 6) len $i t=m$ and for every $i$ such that $i \in \operatorname{dom} i t$ holds $i t(i)=\left(\begin{array}{c}m \\ i-^{\prime} 1\end{array}\right)$. $\left(D^{m+1-^{\prime} i}\right)(x) \cdot\left(D^{i}\right)(y)$.

The functor $\operatorname{LBZ} 2(D, m, x, y)$ yielding a finite sequence of elements of the carrier of $R$ is defined by

(Def. 7) len $i t=m$ and for every $i$ such that $i \in \operatorname{dom} i t \operatorname{holds} i t(i)=\left(\begin{array}{c}m \\ i\end{array}\right)$. $\left(D^{m+1-^{\prime} i}\right)(x) \cdot\left(D^{i}\right)(y)$.

Now we state the propositions:

$$
D\left(\sum \operatorname{LBZ0}(D, n, x, y)\right)=\sum D \cdot(\operatorname{LBZ0}(D, n, x, y))
$$

(18) $\operatorname{LBZ0}(D, m, x, y)=\operatorname{LBZ1}(D, m, x, y)+\operatorname{LBZ2}(D, m, x, y)$.

Proof: Set $p=\operatorname{LBZ1}(D, m, x, y)$. Set $q=\operatorname{LBZ2}(D, m, x, y)$. Set $r=$ $\operatorname{LBZ0}(D, m, x, y)$. For every $k$ such that $1 \leqslant k \leqslant \operatorname{len}(p+q)$ holds $(p+$ q) $(k)=r(k)$. 
(19) $\sum \operatorname{LBZ0}(D, n, x, y)=\sum \operatorname{LBZ} 1(D, n, x, y)+\sum \operatorname{LBZ} 2(D, n, x, y)$. The theorem is a consequence of (18).

(20) $D \cdot(\operatorname{LBZ0}(D, n, x, y))=(\operatorname{LBZ2}(D, n+1, x, y))_{\lceil n+1}+(\operatorname{LBZ} 1(D, n+1, x, y))_{\uparrow 1}$. Proof: Set $p=\operatorname{LBZ2}(D, n+1, x, y)$. Set $q=\operatorname{LBZ1}(D, n+1, x, y)$. Set $r=$ $\operatorname{LBZ0}(D, n, x, y)$. Reconsider $p_{1}=p_{\lceil n+1}$ as a finite sequence of elements of the carrier of $R$. Reconsider $q_{1}=q_{\uparrow 1}$ as a finite sequence of elements of the carrier of $R$. For every $i$ such that $1 \leqslant i \leqslant \operatorname{len} D \cdot r$ holds $(D \cdot r)(i)=$ $\left(p_{1}+q_{1}\right)(i)$.

(21) $\sum D \cdot(\operatorname{LBZ0}(D, n, x, y))=-(\operatorname{LBZ1}(D, n+1, x, y)) / 1+\sum \operatorname{LBZ0}(D, n+$ $1, x, y)-(\operatorname{LBZ} 2(D, n+1, x, y))_{/ n+1}$. The theorem is a consequence of $(20)$ and (19).

(22) $\left.\operatorname{LBZ}(D, n+1, x, y)=\left(\left\langle\left(D^{n+1}\right)(x) \cdot y\right\rangle\right\urcorner \operatorname{LBZ0}(D, n, x, y)\right)^{\frown}\left\langle x \cdot\left(D^{n+1}\right)(y)\right\rangle$. Proof: Set $p=\operatorname{LBZ}(D, n+1, x, y)$. Set $q=\operatorname{LBZ0}(D, n, x, y)$. Set $r=$ $\left(\left\langle\left(D^{n+1}\right)(x) \cdot y\right\rangle^{\frown} q\right)^{\frown}\left\langle x \cdot\left(D^{n+1}\right)(y)\right\rangle$. For every $k$ such that $1 \leqslant k \leqslant \operatorname{len} p$ holds $p(k)=r(k)$.

(23) $\sum\left(\left(\left\langle\left(D^{n+1}\right)(x) \cdot y\right\rangle \frown \operatorname{LBZ0}(D, n, x, y)\right)^{\frown}\left\langle x \cdot\left(D^{n+1}\right)(y)\right\rangle\right)=\left(D^{n+1}\right)(x) \cdot$ $y+\sum \operatorname{LBZ0}(D, n, x, y)+x \cdot\left(D^{n+1}\right)(y)$.

(24) $\quad D\left(\sum \operatorname{LBZ}(D, n+1, x, y)\right)=\sum \operatorname{LBZ}(D, n+2, x, y)$. The theorem is a consequence of $(9),(21),(11),(22)$, and (23).

(25) The Leibniz Formula For POWER OF Derivation:

$\left(D^{n}\right)(x \cdot y)=\sum \operatorname{LBZ}(D, n, x, y)$. The theorem is a consequence of (16), (9), (24), and (15).

\section{Example of Derivation of Polynomial Ring over a Commutative RING}

Let us consider $R$. Let $f$ be a function from $\operatorname{PolyRing}(R)$ into $\operatorname{PolyRing}(R)$ and $p$ be an element of the carrier of PolyRing $(R)$. Observe that the functor $f(p)$ yields an element of the carrier of PolyRing $(R)$. Let $R$ be a ring. The functor $\operatorname{Der1}(R)$ yielding a function from PolyRing $(R)$ into PolyRing $(R)$ is defined by

(Def. 8) for every element $f$ of the carrier of $\operatorname{PolyRing}(R)$ and for every natural number $i$, it $(f)(i)=(i+1) \cdot f(i+1)$.

Let us consider $R$. One can verify that $\operatorname{Der} 1(R)$ is additive.

In the sequel $R$ denotes an integral domain and $f, g$ denote elements of the carrier of PolyRing $(R)$.

Now we state the proposition:

(26) Let us consider an element $f$ of the carrier of $\operatorname{PolyRing}(R)$, and a polynomial $f_{1}$ over $R$. Suppose $f=f_{1}$ and $f_{1}$ is constant. Then $(\operatorname{Der} 1(R))(f)=$ o. $R$. 
Proof: For every element $i$ of $\mathbb{N},(\operatorname{Der} 1(R))(f)(i)=(\mathbf{0} . R)(i)$.

In the sequel $a$ denotes an element of $R$. Now we state the propositions:

(27) Let us consider a natural number $i$, and an element $p$ of the carrier of PolyRing $(R)$. Then $((a \uparrow R) * p)(i)=a \cdot p(i)$.

(28) Let us consider elements $f, g$ of the carrier of PolyRing $(R)$, and an element $a$ of $R$. Suppose $f=a \mid R$. Then $(\operatorname{Der} 1(R))(f \cdot g)=(a \nmid R) *(\operatorname{Der} 1(R))(g)$. Proof: For every natural number $n,(\operatorname{Der} 1(R))(f \cdot g)(n)=((a \nmid R) *$ $(\operatorname{Der1}(R))(g))(n)$.

Let us consider an element $f$ of the carrier of PolyRing $(R)$ and an element $a$ of $R$. Now we state the propositions:

(29) If $f=\operatorname{anpoly}(a, 0)$, then $(\operatorname{Der} 1(R))(f)=\mathbf{0}$. $R$.

Proof: For every element $n$ of $\mathbb{N},(\operatorname{Der} 1(R))(f)(n)=(\mathbf{0} \cdot R)(n)$.

(30) If $f=\operatorname{anpoly}(a, 1)$, then $(\operatorname{Der} 1(R))(f)=\operatorname{anpoly}(a, 0)$.

Proof: For every element $n$ of $\mathbb{N},(\operatorname{Der} 1(R))(f)(n)=(\operatorname{anpoly}(a, 0))(n)$.

(31) Let us consider polynomials $p, q$ over $R$. Suppose $p=\operatorname{anpoly}\left(1_{R}, 1\right)$. Let us consider an element $i$ of $\mathbb{N}$. Then

(i) $(p * q)(i+1)=q(i)$, and

(ii) $(p * q)(0)=0_{R}$.

Proof: For every element $i$ of $\mathbb{N},(p * q)(i+1)=q(i)$. Consider $F_{1}$ being a finite sequence of elements of the carrier of $R$ such that len $F_{1}=0+1$ and $(p * q)(0)=\sum F_{1}$ and for every element $k$ of $\mathbb{N}$ such that $k \in \operatorname{dom} F_{1}$ holds $F_{1}(k)=p\left(k-{ }^{\prime} 1\right) \cdot q\left(0+1-^{\prime} k\right)$.

(32) Let us consider elements $f, g$ of the carrier of $\operatorname{PolyRing}(R)$. Suppose $f=$ $\operatorname{anpoly}\left(1_{R}, 1\right)$. Then $(\operatorname{Der} 1(R))(f \cdot g)=(\operatorname{Der} 1(R))(f) \cdot g+f \cdot(\operatorname{Der} 1(R))(g)$. Proof: Reconsider $d_{1}=(\operatorname{Der} 1(R))(f), d_{2}=(\operatorname{Der} 1(R))(g)$ as a polynomial over $R$. Reconsider $f_{1}=f, g_{1}=g$ as a polynomial over $R$. For every element $i$ of $\mathbb{N},(\operatorname{Der} 1(R))(f \cdot g)(i)=\left(d_{1} * g_{1}+f_{1} * d_{2}\right)(i)$.

(33) Let us consider constant elements $f, g$ of the carrier of $\operatorname{PolyRing}(R)$. Then $(\operatorname{Der} 1(R))(f \cdot g)=(\operatorname{Der} 1(R))(f) \cdot g+f \cdot(\operatorname{Der} 1(R))(g)$. The theorem is a consequence of (29).

(34) Let us consider elements $f, g$ of the carrier of PolyRing $(R)$. Suppose $f$ is constant. Then $(\operatorname{Der} 1(R))(f \cdot g)=(\operatorname{Der} 1(R))(f) \cdot g+f \cdot(\operatorname{Der} 1(R))(g)$. The theorem is a consequence of (29) and (28).

(35) Let us consider elements $x, y$ of the carrier of PolyRing $(R)$. Suppose $x$ is not constant. Then $(\operatorname{Der} 1(R))(x \cdot y)=(\operatorname{Der} 1(R))(x) \cdot y+x \cdot(\operatorname{Der} 1(R))(y)$. Proof: Define $\mathcal{P}$ [natural number] $\equiv$ for every elements $f, g$ of the carrier of PolyRing $(R)$ for every elements $f_{0}, g_{0}$ of the carrier of PolyRing $(R)$ such 
that $f_{0}=f$ and $g_{0}=g$ and $\operatorname{deg} f_{0}-1=\$_{1}$ holds $(\operatorname{Der} 1(R))\left(f_{0} \cdot g_{0}\right)=$ $(\operatorname{Der} 1(R))\left(f_{0}\right) \cdot g_{0}+f_{0} \cdot(\operatorname{Der} 1(R))\left(g_{0}\right)$. For every natural number $k$ such that for every natural number $n$ such that $n<k$ holds $\mathcal{P}[n]$ holds $\mathcal{P}[k]$ by [8, (4)]. For every natural number $n, \mathcal{P}[n]$.

(36) $(\operatorname{Der} 1(R))(f \cdot g)=(\operatorname{Der} 1(R))(f) \cdot g+f \cdot(\operatorname{Der} 1(R))(g)$. The theorem is a consequence of $(35)$ and $(34)$.

Let us consider $R$. Let us observe that $\operatorname{Der} 1(R)$ is derivation.

Now we state the propositions:

(37) Let us consider an element $x$ of $\operatorname{PolyRing}(R)$, and a polynomial $f$ over $R$. If $x=f$, then for every natural number $n, x^{n}=f^{n}$.

Proof: Define $\mathcal{P}$ [natural number $] \equiv x^{\$_{1}}=f^{\$_{1}}$. For every natural number $n$ such that $\mathcal{P}[n]$ holds $\mathcal{P}[n+1]$ by [6, (19)]. For every natural number $n$, $\mathcal{P}[n]$.

(38) Let us consider an element $x$ of PolyRing $(R)$. Suppose $x=\operatorname{anpoly}\left(1_{R}, 1\right)$. Then there exists an element $y$ of $\operatorname{PolyRing}(R)$ such that

(i) $y=\operatorname{anpoly}\left(1_{R}, n\right)$, and

(ii) $(\operatorname{Der} 1(R))\left(x^{n+1}\right)=(n+1) \cdot y$.

The theorem is a consequence of (30), (37), and (7).

From now on $p$ denotes a polynomial over $\mathbb{R}_{\mathrm{F}}$.

Let us consider $p$. The functor $p^{\prime}$ yielding a sequence of $\mathbb{R}_{\mathrm{F}}$ is defined by

(Def. 9) for every natural number $n, i t(n)=p(n+1) \cdot(n+1)$.

Now we state the proposition:

(39) Let us consider an element $p_{0}$ of PolyRing $\left(\mathbb{R}_{\mathrm{F}}\right)$, and a polynomial $p$ over $\mathbb{R}_{\mathrm{F}}$. If $p_{0}=p$, then $p^{\prime}=\left(\operatorname{Der} 1\left(\mathbb{R}_{\mathrm{F}}\right)\right)\left(p_{0}\right)$.

Proof: For every $n,\left(p^{\prime}\right)(n)=\left(\operatorname{Der} 1\left(\mathbb{R}_{\mathrm{F}}\right)\right)\left(p_{0}\right)(n)$.

\section{REFERENCES}

[1] Grzegorz Bancerek, Czesław Byliński, Adam Grabowski, Artur Korniłowicz, Roman Matuszewski, Adam Naumowicz, Karol Pąk, and Josef Urban. Mizar: State-of-the-art and beyond. In Manfred Kerber, Jacques Carette, Cezary Kaliszyk, Florian Rabe, and Volker Sorge, editors, Intelligent Computer Mathematics, volume 9150 of Lecture Notes in Computer Science, pages 261-279. Springer International Publishing, 2015. ISBN 978-3319-20614-1. doi 10.1007/978-3-319-20615-8_17.

[2] Grzegorz Bancerek, Czesław Byliński, Adam Grabowski, Artur Korniłowicz, Roman Matuszewski, Adam Naumowicz, and Karol Pakk. The role of the Mizar Mathematical Library for interactive proof development in Mizar. Journal of Automated Reasoning, 61(1):9-32, 2018. do1 10.1007 /s10817-017-9440-6

[3] Artur Korniłowicz. Differentiability of polynomials over reals. Formalized Mathematics, 25(1):31-37, 2017. doi $10.1515 /$ forma-2017-0002.

[4] Artur Korniłowicz and Christoph Schwarzweller. The first isomorphism theorem and other properties of rings. Formalized Mathematics, 22(4):291-301, 2014. doi 10.2478/forma-20140029. 
[5] Hideyuki Matsumura. Commutative Ring Theory. Cambridge Studies in Advanced Mathematics. Cambridge University Press, 2nd edition, 1989.

[6] Robert Milewski. Fundamental theorem of algebra. Formalized Mathematics, 9(3):461-470, 2001.

[7] Masayoshi Nagata. Theory of Commutative Fields, volume 125 of Translations of Mathematical Monographs. American Mathematical Society, 1985.

[8] Christoph Schwarzweller. On roots of polynomials and algebraically closed fields. Formalized Mathematics, 25(3):185-195, 2017. doi 10.1515/forma-2017-0018.

Accepted March 30, 2021 